\title{
Article \\ AEG-1 Regulates TWIK-1 Expression as an RNA-Binding Protein in Astrocytes
}

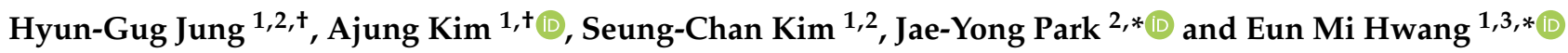 \\ 1 Brain Science Institute, Korea Institute of Science and Technology (KIST), Seoul 02792, Korea; \\ hyungug.jung@einsteinmed.org (H.-G.J.); kitkim819@kist.re.kr (A.K.); 216008@kist.re.kr (S.-C.K.) \\ 2 School of Biosystem and Biomedical Science, College of Health Science, Korea University, Seoul 02841, Korea \\ 3 Division of Bio-Medical Science \& Technology, KIST School, Korea University of Science and Technology, \\ Seoul 02792, Korea \\ * Correspondence: jaeyong68@korea.ac.kr (J.-Y.P.); emhwang@kist.re.kr (E.M.H.); Tel.: +82-2-3290-5637 (J.-Y.P.); \\ +82-2-958-7220 (E.M.H.) \\ + These authors contributed equally to this work.
}

check for updates

Citation: Jung, H.-G.; Kim, A.; Kim, S.-C.; Park, J.-Y.; Hwang, E.M. AEG-1 Regulates TWIK-1 Expression as an RNA-Binding Protein in Astrocytes. Brain Sci. 2021, 11, 85. https://doi. org/10.3390/brainsci11010085

Received: 18 December 2020 Accepted: 8 January 2021 Published: 11 January 2021

Publisher's Note: MDPI stays neutral with regard to jurisdictional clai$\mathrm{ms}$ in published maps and institutional affiliations.

Copyright: (C) 2021 by the authors. Licensee MDPI, Basel, Switzerland. This article is an open access article distributed under the terms and conditions of the Creative Commons Attribution (CC BY) license (https:// creativecommons.org/licenses/by/ $4.0 /)$.

\begin{abstract}
AEG-1, also called MTDH, has oncogenic potential in numerous cancers and is considered a multifunctional modulator because of its involvement in developmental processes and inflammatory and degenerative brain diseases. However, the role of AEG-1 in astrocytes remains unknown. This study aimed to investigate proteins directly regulated by AEG-1 by analyzing their RNA expression patterns in astrocytes transfected with scramble shRNA and AEG-1 shRNA. AEG-1 knockdown down-regulated TWIK-1 mRNA. Real-time quantitative PCR (qPCR) and immunocytochemistry assays confirmed that AEG-1 modulates TWIK-1 mRNA and protein expression. Electrophysiological experiments further revealed that AEG-1 further regulates TWIK-1-mediated potassium currents in normal astrocytes. An RNA immunoprecipitation assay to determine how AEG-1 regulates the expression of TWIK-1 revealed that AEG-1 binds directly to TWIK-1 mRNA. Furthermore, TWIK-1 mRNA stability was significantly increased upon overexpression of AEG-1 in cultured astrocytes $(p<0.01)$. Our findings show that AEG-1 serves as an RNA-binding protein to regulate TWIK-1 expression in normal astrocytes.
\end{abstract}

Keywords: astrocyte; AEG-1; TWIK-1; RNA-binding protein

\section{Introduction}

Astrocytes are the most well-studied glial cells in the brain, and recently, it has been reported that they are involved in pathological mechanisms of CNS diseases such as edema and neurodegeneration [1,2]. In particular, astrogliosis is a common feature of astrocytes that appear in pathological processes, and it is known that astrocyte-elevated gene-1 (AEG1) increases during this process, and it is thus attracting attention as a novel treatment target [3]. AEG-1 was initially identified as an inducible gene in human fetal astrocytes infected with HIV-1 or treated with TNF- $\alpha$ [4]. Many studies of AEG-1 have reported to play a pivotal role in brain cancers as an oncogene, such as glioma and neuroblastoma, and in many other tissue cancers, but not in normal astrocytes [5-10]. AEG-1 is reportedly associated with tumorigenesis and serves as a multifunctional mediator involved in several signalling pathways including PI3K/Akt, NF-кB, Wnt/ $\beta$-catenin, and MAPK [11-13]. Because AEG-1 does not contain any DNA-binding domains, it is believed to regulate these signal transduction pathways through interactions with several transcription factors. Indeed, AEG-1 interacts with transcription factors such as p65, c-Jun, Yin Yang 1, cyclic AMP responsive element-binding protein (CREB), and CREB-binding protein [14-16].

Recently, Hsu et al. reported that AEG-1 is an integral RNA-binding membrane protein in the endoplasmic reticulum (ER) that primarily binds to the coding sequence of ER mRNAs and transmembrane protein-encoding mRNAs $[17,18]$. Although AEG-1 lacks 
a canonical RNA-binding domain, it has been identified as an RNA-binding protein (RBP) by several AEG-1-RNA interactome screens [19-21]. However, its cellular function as an $\mathrm{RBP}$ is still unclear.

TWIK-1, a member of two-pore-domain potassium (K2P) channel family, is highly expressed in normal astrocytes [22]. We first reported that TWIK-1 is a heterodimer with TREK-1 in normal astrocytes, contributing to passive conductance and GPCR-mediated glutamate release [23,24]. Furthermore, we reported that TWIK-1 modulates intrinsic excitability by forming heterodimers with TASK-3 in the dentate gyrus granule cells (DGGC) neurons $[25,26]$. Despite the physiological significance of TWIK-1, few studies have focused on the molecular mechanisms regulating TWIK-1 expression [27].

In this study, we used shRNA to investigate AEG-1 function in normal astrocytes. This study shows that AEG- 1 binds to TWIK- 1 mRNA in the ER in astrocytes and regulates its expression.

\section{Materials and Methods}

\subsection{Plasmids and shRNA}

The cDNA encoding full-length of mouse AEG-1 (GenBank accession no. NM_026002) was synthesized by gBlocks (IDT, Singapore), and mouse TWIK-1 (GenBank accession no. NM_008430) was obtained using a RT-PCR-based Gateway (Invitrogen, Carlsbad, CA, USA). The constructs were subcloned into the pDEST-GFP-N vector by Gateway cloning. The shRNA vector against TWIK-1 or AEG-1 was described in our previous studies [24,28].

\subsection{Primary Astrocyte Culture}

Primary astrocyte cultures are prepared from the cerebral cortices of C57BL/6 mouse pups (P1) as described previously [29]. In brief, the cerebral cortex from 1-day-old C57BL/6 mice were chopped and mechanically disrupted through trituration. The cells obtained were seeded in culture flasks and grown at $37{ }^{\circ} \mathrm{C}$ in a $5 \% \mathrm{CO}_{2}$ atmosphere in DMEM supplemented with $10 \%$ heat-inactivated FBS, $100 \mathrm{U} / \mathrm{mL}$ penicillin-streptomycin. The culture medium was changed after 3-4 days to eliminate debris and other floating cell types. Cells were used and transfected after 5-7 days of culture.

\subsection{RNA Sequencing (RNA-Seq) and Analysis}

Sequencing and analysis were performed by eBiogen (Seoul, Korea). Total RNA of primary cultured astrocytes was isolated as manufacturer (GeneAll, Seoul, Korea). Two samples were prepared for each group. Libraries were prepared from $2 \mu \mathrm{g}$ of total RNA and isolated mRNA. The isolated mRNAs were used for cDNA synthesis and shearing. Indexing was performed using the Illumina index 1-12. Libraries were identified using the Agilent 2100 Bioanalyzer system to evaluate the mean fragment size. Quantification was performed using the library quantification kit using a StepOne RT-qPCR System. Highthroughput sequencing was performed with a $100 \mathrm{bp}$ paired-end protocol using HiSeq 2500 (Illumina, San Diego, CA, USA). mRNA-seq reads were mapped by TopHat software tool to obtain alignment files that were subsequently used to obtain assembling transcripts. mRNA transcript abundance and differential gene expression was analyzed using cufflinks (https:/ / www.genecards.org). Quantile normalized FPKM by EdgeR in R was used to determine expression level of gene regions.

\subsection{RT-PCR and $q P C R$}

Total RNA of primary cultured astrocytes was isolated as manufacturer (GeneAll, Seoul, Korea). For RT-PCR and qPCR, $500 \mathrm{ng}$ of total RNA was used for cDNA preparation with an SensiFAST cDNA Synthesis Kit (BIOLINE, London, UK) according to the manufacturer's protocol. The following RT-PCR primers were used: TWIK-1, 5'-TGCTCTACCTGGTGTTCGG-3' (forward) and 5'-GAGTAGATGATGCAGAAGGC-3' (reverse); TREK-1, 5' CAGAACTCCAA ACCGAGGCT-3' (forward) and 5'-GATGTTTCCAAATCCTATGG-3' (reverse); Actin, 5' - CCCA GATCATGTTTGAGACC-3' (forward) and 5'- TCATGGATGCCACAGGATTC-3' (reverse); 
glyceride-3-phosphate dehydrogenase (GAPDH), 5'-GTCTTCACCACCATGGAGAA-3' (forward) and $5^{\prime}$-GCATGGACTGTGGTCATGAG-3' (reverse). PCR amplification was performed using the 2X TOPsimple DyeMIX-Tenuto (Enzynomics, Daejeon, Korea). qPCR was performed using the SensiFAST Probe Hi-ROX kit (BIOLINE). Primer sets for AEG-1 (Mm.PT.58.10641401), TWIK-1 (Mm.PT.58.11947413) and GAPDH (Mm.PT.39a.1) were purchased from IDT. GAPDH was used as an internal normalization control. Detailed sequence information for each is specified in Table 1 . All experiments were performed in triplicates. The $2^{-\Delta \Delta \mathrm{Ct}}$ method was applied to calculate fold changes in gene expression.

Table 1. qRT-PCR Primers and Probes.

\begin{tabular}{ll}
\hline \multicolumn{1}{c}{ Gene } & \multicolumn{1}{c}{ Sequence } \\
\hline AEG-1 & \\
Forward Primer & $5^{\prime}$-CTATCTTCATCTACCCAGTTCCC-3' \\
Probe & $5^{\prime}$-/56-FAM/CCTAGCTCA/ZEN/GACTGGAATGCACCA/31ABkFQ/-3' \\
Reverse Primer & $5^{\prime}$-GGATGTTAGCCGTAATCAACCT-3' \\
\hline TWIK-1 & \\
Forward Primer & $5^{\prime}$-GCTACAACCAGAAGTTCCGA-3' \\
Probe & $5^{\prime}-/ 56-F A M / C C G A G G A G C / Z E N /$ AGGTAACACGTGAT/3IABkFQ/-3' \\
Reverse Primer & $5^{\prime}$ - TTCAGCTCGTGGAGTTCAC -3' \\
\hline GAPDH & \\
Forward Primer & $5^{\prime}$-GTGGAGTCATACTGGAACATGTAG-3' \\
Probe & $5^{\prime}$-/56-FAM/TGCAAATGG/ZEN/CAGCCCTGGTG/3IABkFQ/-3' \\
Reverse Primer & $5^{\prime}$-AATGGTGAAGGTCGGTGTG-3' \\
\hline
\end{tabular}

\subsection{Immunocytochemistry}

Primary cultured astrocytes were rinsed in PBS and fixed in 4\% PFA for $15 \mathrm{~min}$. Cells were permeabilised 0.1\% Triton X-100 (Sigma-Aldrich, St. Louis, MO, USA) and blocked using $0.1 \%$ BSA for $1.5 \mathrm{~h}$, then incubated with anti-TWIK-1 (Elpisbio, Daejeon, Korea), anti-AEG-1 (40-6500, Invitrogen, Carlsbad, CA, USA), and anti-GFAP (PA1-10004; Thermo Scientific, Waltham, MA, USA) antibodies overnight at $4{ }^{\circ} \mathrm{C}$. This was followed by incubation for $1 \mathrm{~h}$ at room temperature with fluorescence-conjugated and mounted with DAPI-containing gelatin. Images were acquired by a Nikon A1 confocal microscope (Nikon Inc., Tokyo, Japan) at room temperature.

\subsection{Electrophysiology}

Every seventh day after transfection, cells were transferred to a perfusion bath with an artificial extracellular solution containing (in $\mathrm{mM}$ ) $150 \mathrm{NaCl}, 3 \mathrm{KCl}, 2 \mathrm{CaCl} 2,1 \mathrm{MgCl}$, 10 HEPES, 5.5 D-glucose, and 20 sucrose ( $\mathrm{pH} 7.4$ was adjusted with $\mathrm{NaOH}$ ). Glass pipette electrodes (PG150T-15, Harvard Apparatus, Kent, UK) of resistance 3-5 M $\Omega$ were filled with a pseudo-intracellular solution containing (in $\mathrm{mM}$ ) $150 \mathrm{KCl}, 1 \mathrm{CaCl} 2,1 \mathrm{MgCl} 2,5$ EGTA, and 10 HEPES (pH 7.2 was adjusted with $\mathrm{KOH}$ ). Cells were patched using patch clamp in whole cell mode, where current was injected for 1-s voltage ramps from $+50 \mathrm{mV}$ to $-150 \mathrm{mV}$ (from a holding potential of $-60 \mathrm{mV}$ ) using Axopatch 700B amplifier. Data were acquired and digitized using Clampex 10.2 and DigiData 1550B, respectively (Molecular Devices, San Jose, CA, USA). Recordings were carried out at room temperature.

\subsection{Western Blotting}

Samples were lysed using lysis buffer (50 mM HEPES, $0.1 \%$ sodium deoxycholate, $1 \%$ Triton X-100, $1 \mathrm{mM}$ PMSF and 0.1\% SDS) with protease inhibitor cocktail (T\&I, Chuncheon, Korea). The same amount of protein ( $40 \mu \mathrm{g} / \mathrm{lane})$ was added to a polyacrylamide gel $(10 \%)$ for each sample. Proteins separated by SDS-PAGE were electro-transferred to PVDF membranes, and the membranes were blocked using 5\% non-fat milk. After blocking, membrane was incubated with primary antibodies. Antibodies were obtained from the following suppliers: anti-TWIK-1 (4D7, Santa Cruz Biotechnology, Santa Cruz, CA, USA); 
anti-AEG-1 (40-6500, Invitrogen, Carlsbad, CA, USA); and anti- $\beta$-actin (A5541, SigmaAldrich, St. Louis, MO, USA). Membrane was then washed and incubated with HRPconjugated secondary antibodies and incubated at room temperature for $1 \mathrm{~h}$. The blots were detected with ECL Western Blotting Substrate (Thermo Fisher Scientific, Waltham, MA, USA).

\subsection{RNA Immunoprecipitation (RIP) Assay}

RIP assays were conducted using the EZ-Magna RIP Kit (Merck Millipore, Billerica, MA, USA). After transfection with GFP-AEG-1, primary cultured astrocytes were suspended in RIP buffer with mouse IgG or anti-GFP (B-2, Santa Cruz Biotechnology, Dallas, TX, USA). GFP-AEG-1 was immunoprecipitated using protein A/G beads (Santa Cruz Biotechnology), and the amount of TWIK-1 or TREK-1 mRNA in the precipitates was determined through RT-PCR. To increase the sensitivity of the RT-PCR protocol, we performed a second amplification step (30 cycles for TWIK-1, 30 cycles for TREK-1, and 20 cycles for actin) using a $20-\mu \mathrm{L}$ reaction mixture containing $1 \mu \mathrm{L}$ of the first PCR amplification product (20 cycles). The final PCR products were confirmed through sequencing.

\section{9. mRNA Stability Assays}

Cultured astrocytes were treated with $5 \mu \mathrm{g} / \mathrm{mL}$ actinomycin D (Act D, Sigma-Aldrich, St. Louis, MO, USA) for different periods ( $0,2,4,6,8,12$, and $24 \mathrm{~h}$ ). After extracting total RNA from Act D-treated cells, using the RiboX Reagent (GeneAll), RT-PCR was used to confirm the stability of TWIK-1 mRNA.

\subsection{Statistical Analysis}

Data are presented as mean \pm SEM values. Between-group comparisons were performed using Student's t-test, and multiple-group comparisons were performed using ANOVA followed by the Tukey's test for post hoc analysis. For experiments with two independent variables, two-way ANOVA followed by the Tukey's post hoc test was used. Statistical significance was considered at ${ }^{*} p<0.05,{ }^{* *} p<0.01$, and ${ }^{* * *} p<0.001$.

\section{Results}

\subsection{AEG-1 Knockdown Down-Regulates TWIK-1 mRNA in Astrocytes}

To investigate the RNA expression pattern of genes in the presence and absence of AEG-1, we performed RNA-Seq in scramble shRNA- (negative control) and AEG-1 shRNAtransfected astrocytes (AEG-1 knockdown) (Figure 1A). Bioinformatic analysis of RNA-Seq data revealed 132 up-regulated genes and 109 down-regulated genes in AEG-1 knockdown cells rather than in the negative control cells (Figure 1B, Supplementary Table S1). Further, Gene Ontology analysis revealed that most of the down-regulated genes were associated with ion transport pathways (Figure 1C). Thus, we examined the expression patterns of the genes in this GO category (ion transport) and those belonging to the GO category related to the ion channel complex (Figure 1D,E). The generated heat map representing the differences in gene expression indicated that TWIK-1 expression was positively associated with AEG-1 expression. Furthermore, TWIK-1 expression was significantly different in both ion transport and ion channel complex GO categories. qPCR analysis of TWIK-1 further confirmed that its expression was significantly reduced in AEG-1 shRNA-transfected astrocytes than in the negative control cells $(p<0.001)$ (Figure 1F). 
A

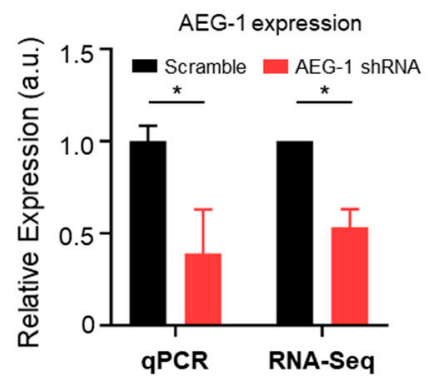

C

\begin{tabular}{|c|c|c|c|}
\hline \multirow[t]{2}{*}{ 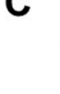 } & \multicolumn{3}{|c|}{$|\mathrm{fc}|>1.2, p<0.05$} \\
\hline & GO Term & $\mathbf{n}$ & P-Value \\
\hline 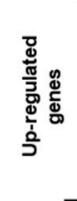 & $\begin{array}{l}\text { Mitotic nuclear division } \\
\text { Cell cycle } \\
\text { Cell division } \\
\text { Mitotic sister chromatic segregation } \\
\text { Chromosome segregation } \\
\text { Centrosome organization } \\
\text { Protein phosphorylation }\end{array}$ & $\begin{array}{c}37 \\
49 \\
39 \\
8 \\
10 \\
7 \\
20\end{array}$ & $\begin{array}{l}4.21 \times 10^{-25} \\
2.05 \times 10^{-23} \\
1.43 \times 10^{-22} \\
1.52 \times 10^{-8} \\
3.18 \times 10^{-6} \\
3.83 \times 10^{-6} \\
3.20 \times 10^{-4} \\
\end{array}$ \\
\hline 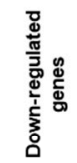 & $\begin{array}{l}\text { Oxidation-reduction process } \\
\text { Positive regulation of macroautophagy } \\
\text { Transport } \\
\text { Lipid metabolic process } \\
\text { Iron ion homeostasis } \\
\text { Ion transport }\end{array}$ & $\begin{array}{c}32 \\
5 \\
56 \\
20 \\
5 \\
21\end{array}$ & $\begin{array}{c}1.87 \times 10^{-5} \\
0.001056402 \\
0.001497374 \\
0.002531672 \\
0.009898212 \\
0.015036541\end{array}$ \\
\hline
\end{tabular}

B - Down-regulated Gene $\bullet$ Up-regulated Gene

(AEG-1 shRNA Scramble) (AEG-1 shRNA Scramble)

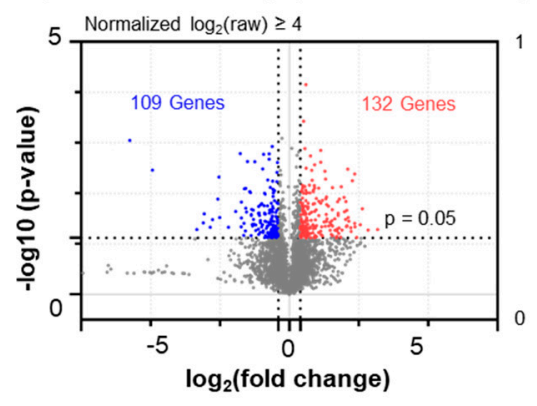

D Go term : Ion transport

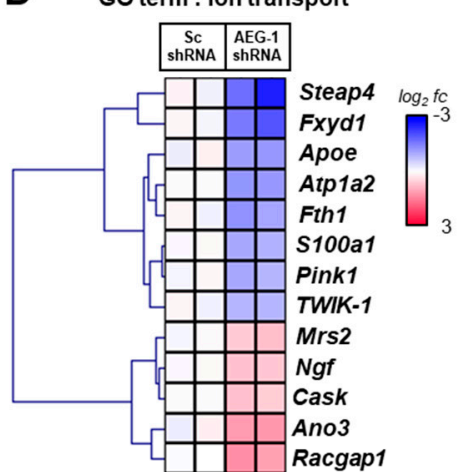

E

Go term : Ion Channel Complex

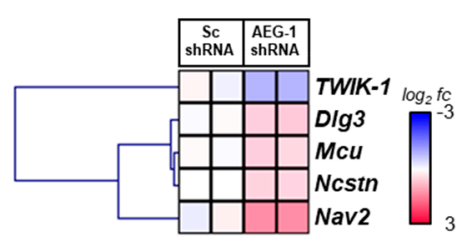

\section{$\mathbf{F}$}

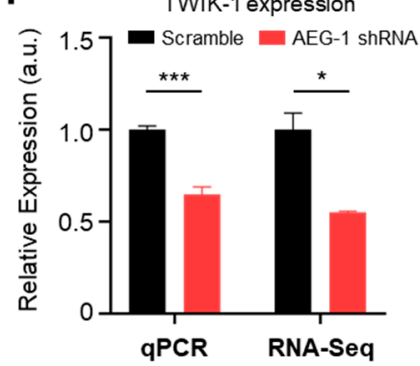

Figure 1. RNA-Seq and bioinformatic analysis of AEG-1 and its effect on TWIK-1 mRNA in cultured astrocytes. (A) Relative expression levels of AEG-1 mRNA in scramble (Sc) shRNA- and AEG-1 shRNA-transfected astrocytes. (B) Volcano plot of $\log _{2}$ (fold change) for genes with values greater than 4 in scramble shRNA- and AEG-1 shRNA-transfected astrocytes. (C) GO Term analysis of differentially expressed genes. (D) Clustered heat map indicating the expression difference between genes in the ion transport GO category (red, up-regulated genes; blue, down-regulated genes). (E) Clustered heat map of genes in the ion channel complex GO category. (F) Relative expression levels of TWIK-1 mRNA in scramble (Sc) shRNAand AEG-1 shRNA-transfected astrocytes. Data are presented as mean \pm SEM. ${ }^{*} p<0.05,{ }^{* * *} p<0.001$.

\subsection{AEG-1 Knockdown Down-Regulates TWIK-1 Protein and TWIK-1-Mediated Potassium Currents in Cultured Astrocytes}

Since AEG-1 knockdown downregulates TWIK-1 mRNA, we investigated whether AEG-1 also regulates the amount and function of the TWIK-1 protein in cultured astrocytes. To determine the amount of endogenous TWIK-1 protein in accordance with AEG-1 expression levels, we performed immunocytochemistry for scramble shRNA- and AEG-1 shRNA-transfected astrocytes. First, we conducted a co-immunostaining experiment with antibodies against astrocyte marker GFAP and AEG-1 to confirm the purity of cultured astrocytes and expression of endogenous AEG-1, and most of the cells expressed both proteins simultaneously (Figure 2A). Figure 2B,C show that AEG-1 shRNA effectively reduced the levels of both proteins, AEG-1 and TWIK-1, compared with scramble shRNA- 
treated cells. The astrocyte has a very dynamic cytoskeleton, and if the amount of protein is large, there is a possibility that the cytoskeleton protein may be saturated [30,31]. So, we confirmed that there was no significant difference in the total amount of protein between samples by showing Ponceau S staining data. We previously reported that TWIK-1 mediates astrocyte potassium currents by forming a heterodimer with TREK-1, one of the other K2P subunits [21]. To assess the influence of AEG-1 on TWIK-1 function, we measured the whole-cell currents in differently treated astrocytes. Overexpression of AEG-1 and TWIK-1 shRNA, alone, and in combination, significantly reduced potassium currents in primary cultures of astrocytes ( $p<0.01$ and $p<0.001$, respectively) (Figure 2D). The current density was then normalized at $+50 \mathrm{mV}$ and $-150 \mathrm{mV}$ compared to the scramble shRNA-transfected conditions (Figure 2E). These data indicate that suppression of AEG-1 decreases both the amount and function of TWIK-1 in cultured astrocytes.

A
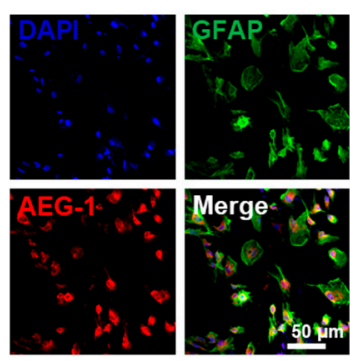

C

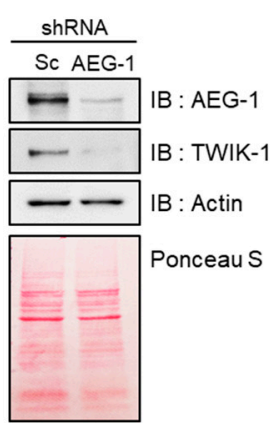

B

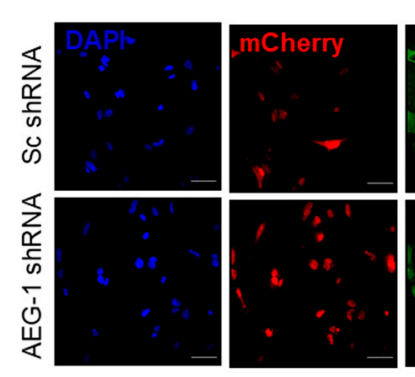

D
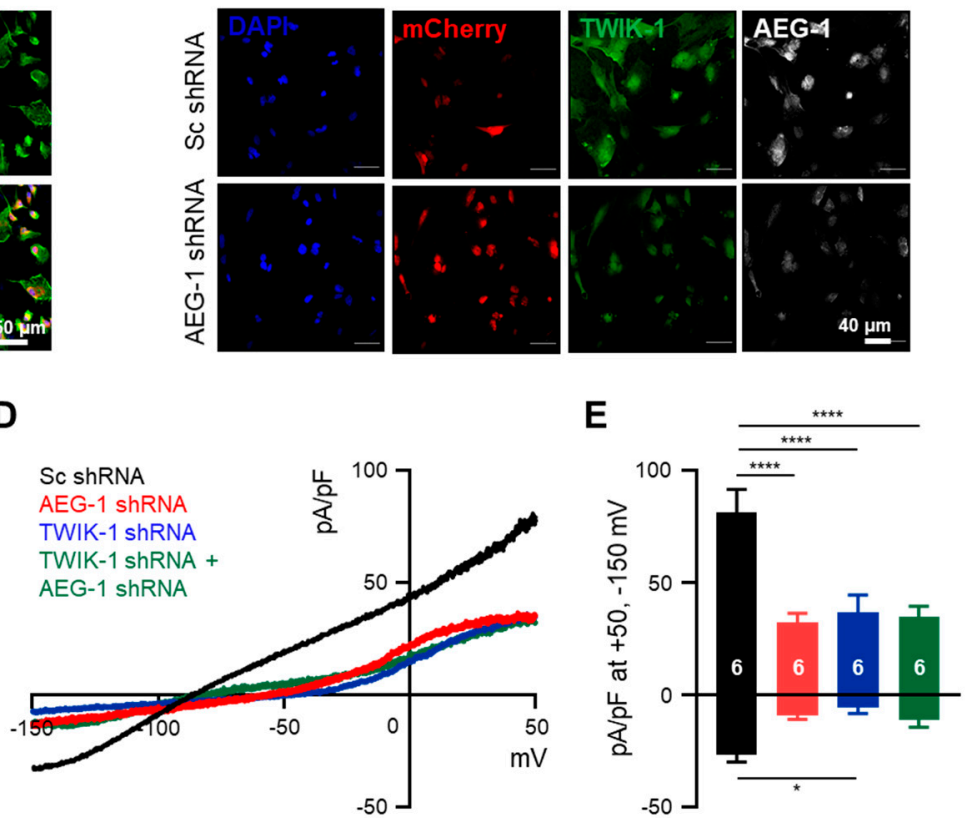

Figure 2. AEG-1 knockdown reduces TWIK-1 protein levels and TWIK-1-mediated potassium currents in primary cultured astrocytes. (A) Immunofluorescence staining of cultured astrocytes using AEG-1 and GFAP, an astrocyte marker. (B) Immunofluorescence staining of TWIK-1 and AEG-1 in transfected astrocyte. Knockdown of the AEG-1 inhibits the levels of TWIK-1. Scale bar, $40 \mu \mathrm{m}$. The shRNA vector contains a shRNA sequence under the control of U6 promoter, with mCherry co-expression under a CMV promoter. (C) Knockdown of the AEG-1 in primary cultured astrocytes. Immunoblot data show the decreased AEG-1 and TWIK-1 expression. (D) Representative whole-cell I-V curves of astrocytes overexpressing Sc shRNA (black), AEG-1 shRNA (red), TWIK-1 shRNA (blue), and AEG-1 and TWIK-1 shRNAs (green). (E) Pooled data for whole-cell current amplitudes in AEG-1 knockdown, TWIK-1 knockdown, and AEG-1 and TWIK-1 double knockdown astrocytes. All values are presented as mean \pm SEM. ${ }^{*} p<0.05,{ }^{* * * *} p<0.0001$. Two-way ANOVA followed by the Tukey's post hoc.

\subsection{AEG-1 Overexpression Up-Regulates TWIK-1 mRNA and Protein and Astrocytic Potassium Currents in Cultured Astrocytes}

Considering the suppression of TWIK-1 expression by AEG-1 inhibition, we tested if TWIK-1 expression can be directly increased through AEG-1 overexpression. As expected, AEG-1 overexpression significantly up-regulated TWIK-1 mRNA and protein levels in cultured astrocytes $(p<0.001)$ (Figure 3A,B). Whole-cell current recordings revealed that the astrocytic potassium currents were also significantly increased upon AEG-1 overexpression 
$(p<0.05)$ (Figure 3C,D). These data show that AEG-1 overexpression enhances TWIK-1 expressions and astrocytic potassium currents in primary cultured astrocytes.
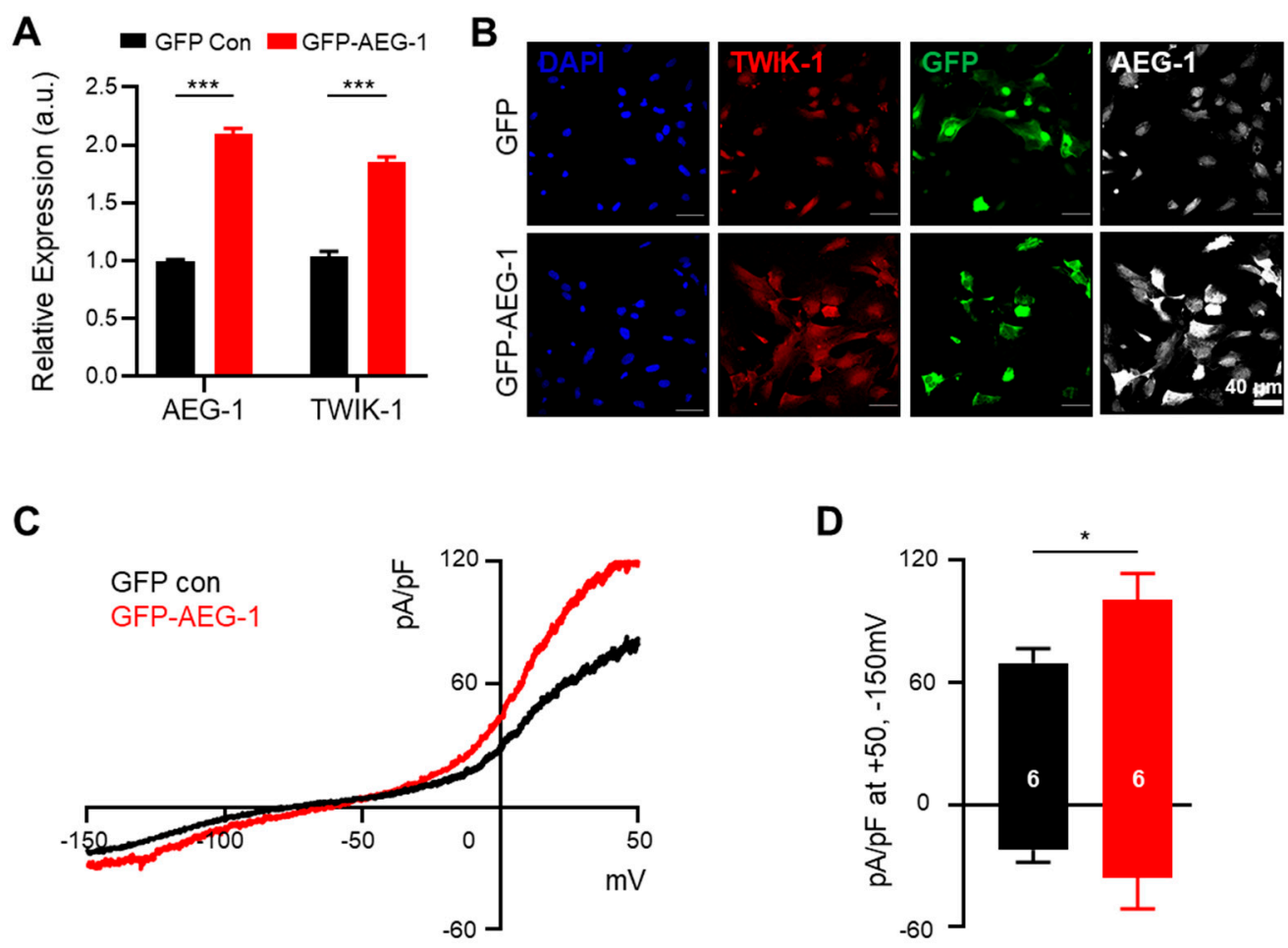

Figure 3. AEG-1 overexpression enhances TWIK-1 mRNA and protein levels and astrocytic potassium currents in cultured astrocytes. (A) Overexpression of AEG-1 increases TWIK-1 mRNAs (B) Immunofluorescence staining of TWIK-1 and AEG-1 in transfected astrocytes. AEG-1 overexpression increases expression levels of TWIK-1. Scale bar, $40 \mu \mathrm{m}$. (C) Representative traces from transfected astrocytes with GFP (control)- and GFP-AEG-1. (D) Bar graph showing potassium currents averaged from results in $(\mathbf{C})$ at $+50 \mathrm{mV}$ and $-150 \mathrm{mV}$. The number on each bar indicates $\mathrm{n}$ for each condition. All values are presented as mean \pm SEM. ${ }^{*} p<0.05,{ }^{* * *} p<0.001$. P-values were obtained from the Student's t-test.

\subsection{AEG-1 Is an RNA-Binding Protein That Enhances TWIK-1 mRNA Stability}

We investigated how AEG-1 regulates TWIK-1 expression. We recently reported that AEG-1 potentially up-regulates another astrocytic K2P channel, namely, TREK-1 [25]. As a follow-up study, we performed a TREK-1 promoter assay to examine the regulatory mechanism; however, AEG-1 did not display any effects. We hypothesized that, since AEG-1 is an ER RBP [17], it may also bind to TWIK-1 mRNA and regulate its stability. Thus, we investigated whether AEG-1 can bind to TWIK-1 mRNA, through RNA immunoprecipitation, followed by RT-PCR (Figure 4A). As shown in Figure 4B, AEG-1 bound to endogenous TWIK-1 mRNA, but not to actin mRNA (control), in cultured astrocytes. We then tested the stability of TWIK-1 mRNA using the RNA polymerase inhibitor Act $\mathrm{D}$, which is widely used to confirm the stability of existing mRNA by preventing new RNA synthesis [32]. Incubation of cultured astrocytes with Act D for $8 \mathrm{~h}$ significantly down-regulated TWIK-1 mRNA in comparison with the control (GAPDH) (Figure 4C,D). To determine the effect of AEG-1 on the stability of TWIK-1 mRNA, we performed qPCR in GFP- and GFP-AEG-1-transfected astrocytes after 8-h incubation with Act D. The stability of TWIK-1 mRNA was significantly increased in GFP-AEG-1-transfected astrocytes compared to that in the GFP-transfected sample $(p<0.01)$ (Figure 4E), indicating that AEG-1 acts as an RBP that regulates TWIK-1 mRNA stability. 
A

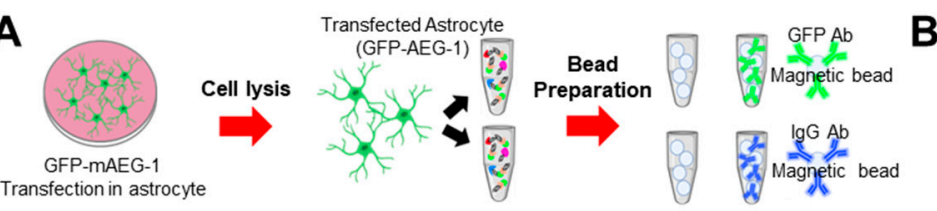

B
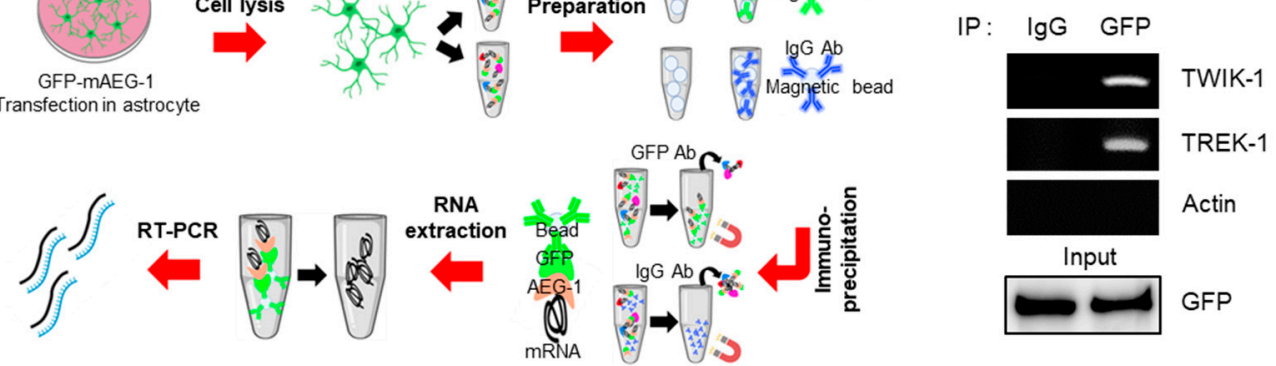

C

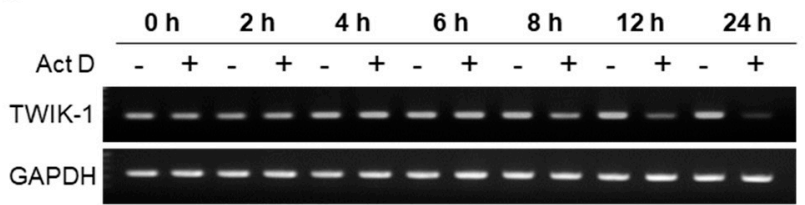

D

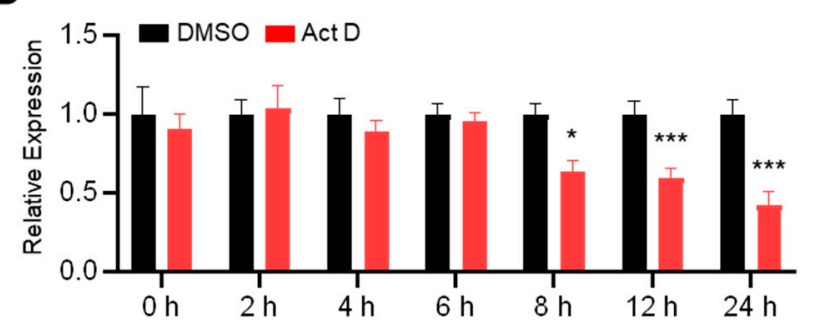

$\mathbf{E}$

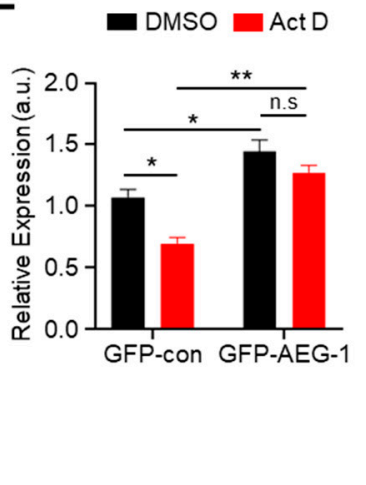

Figure 4. AEG-1 binds with TWIK-1 mRNA and enhances its mRNA stability. (A) Scheme of the RIP assay. (B) Immunoprecipitation of TWIK-1, TREK-1, and actin (negative control). RIP assay was performed on transfected astrocytes with GFP-AEG-1. Immunoprecipitation was performed with mouse IgG and GFP antibodies. Total RNA was purified, and TWIK-1 and TREK-1 mRNAs were detected by RT-PCR. Five percent of the total lysate was used as an input control. Actin was used as the negative control. Primary cultured astrocytes were incubated with Act D ( $5 \mu \mathrm{g} / \mathrm{mL})$, and total RNA was harvested after various incubation times. TWIK-1 mRNA levels were determined using RT-PCR (C) and normalized to the GAPDH (D). Data show the mean \pm SEM. ${ }^{*} p<0.05,{ }^{* * *} p<0.001$ (Student's t-test). (E) Relative expression levels of TWIK-1 mRNA after additional $8 \mathrm{~h}$ incubation with Act D $(5 \mu \mathrm{g} / \mathrm{mL})$ in transfected astrocytes with GFP-AEG-1. Total RNA was harvested from astrocytes after eight hours of incubation. Data are presented as mean $\pm \mathrm{SEM}$. ${ }^{*} p<0.05,{ }^{* *} p<0.01$. P-values were obtained from the two-way ANOVA with Tukey's multiple comparison post hoc test.

\section{Discussion}

Most studies of AEG-1 have focused on its role in cancer and neuronal diseases. However, little is known about the function of AEG-1 in normal astrocytes. Recently, AEG1 has been reported as an RBP that plays an essential role in the post-transcriptional gene expression [17]. RNA-binding proteins bind both RNAs and proteins and are thus involved in multiple biological processes, such as pre-mRNA splicing, modification, transport, transcription, translation, and stability [33-35].

Here, we unveiled that AEG-1, serves as an RNA-binding protein, regulates TWIK1 expression in normal astrocytes. Furthermore, binding of AEG-1 to TWIK-1 mRNA prevented the mRNA from degradation in normal astrocytes, which led to the preservation of the function of TWIK-1 transcript and protein.

Since the expression of AEG-1 is up-regulated in cancer cells and increases with cancer progression, it is considered a therapeutic target for brain cancers. In contrast, the expression of AEG-1 is relatively low in healthy brains, where it is believed to exhibit neuroprotective effects. Indeed, in some degenerative neurological diseases, such as ALS 
and Parkinson's disease, a decrease in neuronal AEG-1 has been reported. In addition, the up-regulation of AEG-1 led to neuronal protection in vivo [36,37].

We previously reported that AEG-1 regulates TREK-1, the latter of two pore potassium channels that are highly expressed in astrocytes. During acute hypoxia, hypoxia-inducing factor $1 \alpha(\mathrm{HIF}-1 \alpha)$ levels are increased and directly induce AEG-1 expression, which in turn up-regulates TREK-1 in astrocytes [28]. The present results show that AEG-1 upregulates TWIK-1 in astrocytes. Based on previous and current studies, astrocytic TWIK-1 and TREK-1 increased by AEG-1 potentially contribute to the protection of excitatory neuronal death by absorbing extracellular potassium ions secreted from activated neurons under pathological conditions. Thus, AEG-1 inhibition should be carefully reviewed as a therapeutic strategy for cancer treatment in future studies.

In this study, since gene profiling was analyzed using only 2D cultured astrocytes, there may be limitations in that there is variability in 3D cultured astrocytes and genetic and protein profiling. Future studies may utilize 3D self-constructing models and organon-a-chip platforms for advanced imaging that can apply spatial transcriptomes along with other imaging modalities such as extended microscopes to overcome previous limitations. Furthermore, the use of humanized models such as human iPSC-derived astrocytes and advanced spatial transcriptomic techniques such as MERFISH [38] are expected to provide more useful information on disease treatment.

\section{Conclusions}

In summary, we identified that AEG-1 has vital role to play in novel regulator of TWIK-1 in normal astrocytes. AEG-1 stimulates TWIK-1 expression and TWIK-1-mediated astrocytic potassium currents by stabilizing TWIK-1 mRNA. This represents a new AEG1-mediated mechanism by which TWIK-1 is regulated under physiological or certain pathological conditions.

Supplementary Materials: The following are available online at https:/ /www.mdpi.com/2076-342 5/11/1/85/s1, Table S1: Raw data.

Author Contributions: H.-G.J. and A.K. designed the experiments and analysed and interpreted the data. H.-G.J., A.K., and S.-C.K. performed the experiments. J.-Y.P. and E.M.H. helped prepare the manuscript. All authors have read and agreed to the published version of the manuscript.

Funding: This work was supported by the National Research Foundation (NRF) of Korea (NRF2017R1A2B3012502 and 2020R1A2C2010650).

Institutional Review Board Statement: This study was performed in accordance with the institutional guidelines of the Korea Institute of Science and Technology (KIST, Seoul, Korea, KIST-2019-044, 2019-06-01).

Data Availability Statement: The data presented in this study are available on request from the corresponding author.

Conflicts of Interest: The authors declare no conflict of interest.

\section{References}

1. Kitchen, P.; Salman, M.M.; Halsey, A.M.; Clarke-Bland, C.; MacDonald, J.A.; Ishida, H.; Vogel, H.J.; Almutiri, S.; Logan, A.; Kreida, S.; et al. Targeting Aquaporin-4 Subcellular Localization to Treat Central Nervous System Edema. Cell 2020, 181, 784-799.e19. [CrossRef]

2. Li, K.; Li, J.; Zheng, J.; Qin, S. Reactive Astrocytes in Neurodegenerative Diseases. Aging Dis. 2019, 10, 664. [CrossRef] [PubMed]

3. Vartak-Sharma, N.; Ghorpade, A. Astrocyte elevated gene-1 regulates astrocyte responses to neural injury: Implications for reactive astrogliosis and neurodegeneration. J. Neuroinflamm. 2012, 9. [CrossRef] [PubMed]

4. Kang, D.C.; Su, Z.Z.; Sarkar, D.; Emdad, L.; Volsky, D.J.; Fisher, P.B. Cloning and characterization of HIV-1-inducible astrocyte elevated gene-1, AEG-1. Gene 2005, 353, 8-15. [CrossRef] [PubMed]

5. $\quad$ Emdad, L.; Sarkar, D.; Lee, S.G.; Su, Z.Z.; Yoo, B.K.; Dash, R.; Yacoub, A.; Fuller, C.E.; Shah, K.; Dent, P.; et al. Astrocyte elevated gene-1: A novel target for human glioma therapy. Mol. Cancer Ther. 2010, 9, 79-88. [CrossRef]

6. Liu, L.; Wu, J.; Ying, Z.; Chen, B.; Han, A.; Liang, Y.; Song, L.; Yuan, J.; Li, J.; Li, M. Astrocyte elevated gene-1 upregulates matrix metalloproteinase-9 and induces human glioma invasion. Cancer Res. 2010, 70, 3750-3759. [CrossRef] 
7. $\quad$ Lee, S.G.; Jeon, H.Y.; Su, Z.Z.; Richards, J.E.; Vozhilla, N.; Sarkar, D.; Van Maerken, T.; Fisher, P.B. Astrocyte elevated gene-1 contributes to the pathogenesis of neuroblastoma. Oncogene 2009, 28, 2476-2484. [CrossRef]

8. Robertson, C.L.; Srivastava, J.; Rajasekaran, D.; Gredler, R.; Akiel, M.A.; Jariwala, N.; Siddiq, A.; Emdad, L.; Fisher, P.B.; Sarkar, D. The role of AEG-1 in the development of liver cancer. Hepat. Oncol. 2015, 2, 303-312. [CrossRef]

9. Li, M.; Dai, Y.; Wang, L.; Li, L. Astrocyte elevated gene-1 promotes the proliferation and invasion of breast cancer cells by activating the Wnt/beta-catenin signaling pathway. Oncol. Lett. 2017, 13, 2385-2390. [CrossRef]

10. Wu, S.; Zhang, Z.; Wu, D.; Chen, H.; Qian, X.; Wang, X.; Huang, W. AEG-1 promotes the growth of gastric cancer through the upregulation of eIF4E expression. OncoTargets Ther. 2019, 12, 5887-5895. [CrossRef]

11. Zhang, F.; Yang, Q.; Meng, F.; Shi, H.; Li, H.; Liang, Y.; Han, A. Astrocyte elevated gene-1 interacts with beta-catenin and increases migration and invasion of colorectal carcinoma. Mol. Carcinog. 2013, 52, 603-610. [CrossRef] [PubMed]

12. Shi, X.; Wang, X. The role of MTDH/AEG-1 in the progression of cancer. Int. J. Clin. Exp. Med. 2015, 8, 4795-4807. [PubMed]

13. Yoo, B.K.; Emdad, L.; Lee, S.G.; Su, Z.Z.; Santhekadur, P.; Chen, D.; Gredler, R.; Fisher, P.B.; Sarkar, D. Astrocyte elevated gene-1 (AEG-1): A multifunctional regulator of normal and abnormal physiology. Pharmacol. Ther. 2011, 130, 1-8. [CrossRef] [PubMed]

14. Emdad, L.; Sarkar, D.; Su, Z.Z.; Randolph, A.; Boukerche, H.; Valerie, K.; Fisher, P.B. Activation of the nuclear factor kappaB pathway by astrocyte elevated gene-1: Implications for tumor progression and metastasis. Cancer Res. 2006, 66, 1509-1516. [CrossRef] [PubMed]

15. Liu, L.; Guan, H.; Li, Y.; Ying, Z.; Wu, J.; Zhu, X.; Song, L.; Li, J.; Li, M. Astrocyte Elevated Gene 1 Interacts with Acetyltransferase p300 and c-Jun To Promote Tumor Aggressiveness. Mol. Cell. Biol. 2017, 37. [CrossRef]

16. Lee, S.G.; Kim, K.; Kegelman, T.P.; Dash, R.; Das, S.K.; Choi, J.K.; Emdad, L.; Howlett, E.L.; Jeon, H.Y.; Su, Z.Z.; et al. Oncogene AEG-1 promotes glioma-induced neurodegeneration by increasing glutamate excitotoxicity. Cancer Res. 2011, 71, 6514-6523. [CrossRef]

17. Hsu, J.C.C.; Reid, D.W.; Hoffman, A.M.; Sarkar, D.; Nicchitta, C.V. Oncoprotein AEG-1 is an endoplasmic reticulum RNA-binding protein whose interactome is enriched in organelle resident protein-encoding mRNAs. RNA 2018, 24, 688-703. [CrossRef]

18. Bethune, J.; Jansen, R.P.; Feldbrugge, M.; Zarnack, K. Membrane-Associated RNA-Binding Proteins Orchestrate OrganelleCoupled Translation. Trends Cell Biol. 2019, 29, 178-188. [CrossRef]

19. Kwon, S.C.; Yi, H.; Eichelbaum, K.; Fohr, S.; Fischer, B.; You, K.T.; Castello, A.; Krijgsveld, J.; Hentze, M.W.; Kim, V.N. The RNA-binding protein repertoire of embryonic stem cells. Nat. Struct. Mol. Biol. 2013, 20, 1122-1130. [CrossRef]

20. Jagannathan, S.; Hsu, J.C.; Reid, D.W.; Chen, Q.; Thompson, W.J.; Moseley, A.M.; Nicchitta, C.V. Multifunctional roles for the protein translocation machinery in RNA anchoring to the endoplasmic reticulum. J. Biol. Chem. 2014, 289, 25907-25924. [CrossRef]

21. Castello, A.; Fischer, B.; Frese, C.K.; Horos, R.; Alleaume, A.M.; Foehr, S.; Curk, T.; Krijgsveld, J.; Hentze, M.W. Comprehensive Identification of RNA-Binding Domains in Human Cells. Mol. Cell 2016, 63, 696-710. [CrossRef] [PubMed]

22. Cahoy, J.D.; Emery, B.; Kaushal, A.; Foo, L.C.; Zamanian, J.L.; Christopherson, K.S.; Xing, Y.; Lubischer, J.L.; Krieg, P.A.; Krupenko, S.A.; et al. A transcriptome database for astrocytes, neurons, and oligodendrocytes: A new resource for understanding brain development and function. J. Neurosci. 2008, 28, 264-278. [CrossRef] [PubMed]

23. Woo, D.H.; Han, K.S.; Shim, J.W.; Yoon, B.E.; Kim, E.; Bae, J.Y.; Oh, S.J.; Hwang, E.M.; Marmorstein, A.D.; Bae, Y.C.; et al. TREK-1 and Best1 channels mediate fast and slow glutamate release in astrocytes upon GPCR activation. Cell 2012, 151, 25-40. [CrossRef] [PubMed]

24. Hwang, E.M.; Kim, E.; Yarishkin, O.; Woo, D.H.; Han, K.S.; Park, N.; Bae, Y.; Woo, J.; Kim, D.; Park, M.; et al. A disulphide-linked heterodimer of TWIK-1 and TREK-1 mediates passive conductance in astrocytes. Nat. Commun. 2014, 5, 3227. [CrossRef] [PubMed]

25. Yarishkin, O.; Lee, D.Y.; Kim, E.; Cho, C.H.; Choi, J.H.; Lee, C.J.; Hwang, E.M.; Park, J.Y. TWIK-1 contributes to the intrinsic excitability of dentate granule cells in mouse hippocampus. Mol. Brain 2014, 7, 80. [CrossRef]

26. Choi, J.H.; Yarishkin, O.; Kim, E.; Bae, Y.; Kim, A.; Kim, S.C.; Ryoo, K.; Cho, C.H.; Hwang, E.M.; Park, J.Y. TWIK-1/TASK-3 heterodimeric channels contribute to the neurotensin-mediated excitation of hippocampal dentate gyrus granule cells. Exp. Mol. Med. 2018, 50, 1-13. [CrossRef]

27. Saito, K.; Moore, R.; Negishi, M. Nuclear receptor CAR specifically activates the two-pore K+ channel Kcnk1 gene in male mouse livers, which attenuates phenobarbital-induced hepatic hyperplasia. Toxicol. Sci. 2013, 132, 151-161. [CrossRef]

28. Kim, A.; Jung, H.G.; Kim, S.C.; Choi, M.; Park, J.Y.; Lee, S.G.; Hwang, E.M. Astrocytic AEG-1 regulates expression of TREK-1 under acute hypoxia. Cell Biochem. Funct. 2020, 38, 167-175. [CrossRef]

29. Lee, C.J.; Mannaioni, G.; Yuan, H.; Woo, D.H.; Gingrich, M.B.; Traynelis, S.F. Astrocytic control of synaptic NMDA receptors. J. Physiol. 2007, 581, 1057-1081. [CrossRef]

30. Taylor, S.C.; Posch, A. The design of a quantitative western blot experiment. BioMed Res. Int. 2014, 2014. [CrossRef]

31. Pillai-Kastoori, L.; Schutz-Geschwender, A.R.; Harford, J.A. A systematic approach to quantitative Western blot analysis. Anal. Biochem. 2020, 593. [CrossRef] [PubMed]

32. Perry, R.P.; Kelley, D.E. Inhibition of Rna Synthesis by Actinomycin-D—Characteristic Dose-Response of Different Rna Species. J. Cell. Physiol. 1970, 76, 127-139. [CrossRef] [PubMed]

33. Keene, J.D. RNA regulons: Coordination of post-transcriptional events. Nat. Rev. Genet. 2007, 8, 533-543. [CrossRef] [PubMed]

34. Glisovic, T.; Bachorik, J.L.; Yong, J.; Dreyfuss, G. RNA-binding proteins and post-transcriptional gene regulation. FEBS Lett. 2008, 582, 1977-1986. [CrossRef] 
35. Halbeisen, R.E.; Galgano, A.; Scherrer, T.; Gerber, A.P. Post-transcriptional gene regulation: From genome-wide studies to principles. Cell. Mol. Life Sci. 2008, 65, 798-813. [CrossRef]

36. Yin, X.; Ren, M.; Jiang, H.; Cui, S.; Wang, S.; Jiang, H.; Qi, Y.; Wang, J.; Wang, X.; Dong, G.; et al. Downregulated AEG-1 together with inhibited PI3K/Akt pathway is associated with reduced viability of motor neurons in an ALS model. Mol. Cell. Neurosci. 2015, 68, 303-313. [CrossRef]

37. Leem, E.; Kim, H.J.; Choi, M.; Kim, S.; Oh, Y.S.; Lee, K.J.; Choe, Y.S.; Um, J.Y.; Shin, W.H.; Jeong, J.Y.; et al. Upregulation of neuronal astrocyte elevated gene-1 protects nigral dopaminergic neurons in vivo. Cell Death Dis. 2018, 9, 449. [CrossRef]

38. Xia, C.; Fan, J.; Emanuel, G.; Hao, J.; Zhuang, X. Spatial transcriptome profiling by MERFISH reveals subcellular RNA compartmentalization and cell cycle-dependent gene expression. Proc. Natl. Acad. Sci. USA 2019, 116, 19490-19499. [CrossRef] 